\title{
Calculation of radial distribution functions for molecules in excited vibrational states
}

\author{
M. A. Kacner and L. S. Bartell \\ Department of Chemistry, University of Michigan, Ann Arbor, Michigan 48109 \\ (Received 14 March 1979; accepted 5 April 1979) \\ It is shown how to reduce $N$-dimensional probability densities calculated from vibrational wave functions \\ of polyatomic molecules to one-dimensional radial distribution functions. Such distributions are, in \\ principle, derivable from diffraction experiments. In this initial treatment, anharmonicity and \\ nonlinearities in transformations between normal coordinates and internuclear distances are neglected. \\ Illustrative calculations are given for $\mathrm{H}_{2} \mathrm{O}, \mathrm{CF}_{3} \mathrm{Cl}$, and $\mathrm{SF}_{6}$ in various vibrational states or thermal \\ distributions among vibrational states. Possible applications are briefly discussed.
}

\section{INTRODUCTION}

Infrared lasers have greatly facilitated the study of molecular systems in states far from thermal equilibrium. They have made possible fruitful investigations of intra- and intermolecular energy transfer ${ }^{1}$ and, indeed, have led to a new field of laser-induced processes. ${ }^{2}$ Since some of these processes are difficult to follow by the techniques currently employed, it seemed worthwhile to consider applying gas - phase electron diffraction analysis to molecules subjected to intense laser radiation. In the electron diffraction method, the information derivable, in principle, is the radial distribution of internuclear distances averaged over the molecular ensemble. A large body of literature exists describing the influence of molecular vibrations on the radial distribution function $p(r)$ for samples in thermal equilibrium. ${ }^{3-5}$ As far as we know, however, no description exists for the treatment of molecules in arbitrary vibrational states or arbitrary distributions among vibrational states. Therefore, the present investigation is addressed to the development of such a treatment.

\section{THEORY}

\section{A. Level of approximation}

If a general expression were available to represent the vibrational wave function $\psi\left(Q_{1}, \ldots, Q_{N}\right)$ for a molecule of interest, it would be a tedious but straightforward exercise (as we shall show) to reduce the multidimensional probability density $|\psi|^{2}$ to the one-dimensional radial distribution function $p(r)$. Two complications arise immediately, however. The first of these is that there is a nonlinear transformation between the normal coordinates $Q_{k}$ and the internuclear distances $r_{i}$, between any pair of atoms $i$ and $j .{ }^{6}$ For vibrations of very small amplitude, only the linear terms are important, of course, but for actual molecular vibrations, particularly in the case of excited states, higher-order terms are necessary for a precise treatment. These corrections are derivable from geometric considerations. The second complication is far less tractable. It is that normal coordinate theory, itself, can provide the required wave functions simply only for the fictional case of molecules undergoing infinitesimal vibrations. For real molecules, effects of anharmonicity are significant ${ }^{5-8}$ but known accurately for only a handful of polyatomic molecules. Since the ill-characterized effects of an- harmonicity are of the same magnitude as, or larger than, the effects of the nonlinear transformation between the normal coordinates and interatomic distances, it is reasonable in this first attack to neglect both of the aforementioned complications. Results, while slightly distorted, reveal the essential nature of the influence of vibrational state upon the observable $p(r)$.

\section{B. Relation between normal vibrations and $r_{i j}$}

For a polyatomic molecule executing harmonic vibrations in a given stationary state, the vibrational wave function $\psi$ can be written as $^{9}$

$$
\psi=\psi_{v 1}\left(Q_{1}\right) \psi_{v 2}\left(Q_{2}\right) \cdots \psi_{v N}\left(Q_{N}\right),
$$

where the individual functions $\psi_{v k}\left(Q_{k}\right)$ for each of the $N$ normal coordinates $Q_{k}$ are the well known SHO eigenfunctions associated with the quantum states $v_{k}$. Accordingly, the expression

$$
P_{Q}\left(Q_{\mathfrak{t}}, \ldots, Q_{N}\right) d Q_{1} \cdots d Q_{N}=\prod_{k}\left|\psi_{v k}\left(Q_{k}\right)\right|^{2} d Q_{k}
$$

represents the probability of finding the molecule displaced from equilibrium along its $k$ th normal coordinate between $Q_{k}$ and $Q_{k}+d Q_{k}$, along its $l$ th normal coordinate between $Q_{l}$ and $Q_{l}+d Q_{l}$, and so on. For a given pair of atoms $i$ and $j$, we seek the radial distribution function $P_{i j}(r)$ for which $P_{i j}(r) d r$ is the probability of finding the internuclear distance between $r$ and $r+d r$. This requires that we relate the internuclear distances $r$ (denoted as $r_{i j}$ in the following) to the normal coordinates. To this end, internuclear displacements can be expressed by the Taylor series expansion

$$
\begin{aligned}
\Delta r_{i j} & =r_{i j}-\left(r_{e}\right)_{i j} \\
& =\sum_{k}\left(\mathcal{L}_{k}\right)_{i j} Q_{k}+\frac{1}{2} \sum_{k} \sum_{i}\left(\mathcal{L}_{k l}\right)_{i j} Q_{k} Q_{l}+\cdots,
\end{aligned}
$$

where, as explained in Sec. II A, we retain only the linear terms and where the coefficients ${ }^{10}$

$$
\left(\mathcal{L}_{k}\right)_{i j}=\left(m_{i}^{-1 / 2} \mathbf{l}_{i k}-m_{j}^{-1 / 2} \mathbf{l}_{j k}\right) \cdot \mathbf{r}_{i j} /\left|\boldsymbol{r}_{i j}\right|
$$

can be derived from a normal coordinate analysis. In Eq. (4), $m_{i}$ and $1_{i k}$ are, respectively, the mass and the mass-weighted amplitude eigenvector of atom $i$ for the $k$ th mode. It is convenient to re-express Eq. (3) as

$$
\Delta r_{i j}=\sum_{k}\left(x_{k}\right)_{i j}
$$


where the variable $\left(x_{k}\right)_{i j}=\left(\mathscr{L}_{k}\right)_{i j} Q_{k}$ is the contribution of mode $k$ to $\Delta r_{i j}$.

It remains to be shown how to reduce the probability density $P_{Q}\left(Q_{1}, \ldots, Q_{N}\right)$ of Eq. (2) to the $i, j$ radial distribution function $P_{i j}(r)$. For expedience, we shall henceforth use $P_{i j}(r)=P_{i j}\left(r_{e}+\Delta r\right)$ and $P_{i j}(\Delta r)$ interchangeably without comment, letting the meaning be inferred from the context. In order to make the reduction procedure plain, we shall first carry it out explicitly in the next section for a two dimensional illustrative case. We shall then treat the general case of an $N$ dimensional harmonic oscillator in the following section.

\section{Reduction of two dimensional density function}

Although it is not necessary in the formal mathematical treatment to specify the physical system involved, it may make the exposition clearer if a concrete example is suggested. Let us suppose that the normal coordinates $Q_{1}$ and $Q_{2}$ pertain to the symmetric and asymmetric stretching modes $\nu_{1}$ and $\nu_{3}$, respectively, of a (nonbending) linear triatomic molecule. Then, for the ground state of the molecule, the probability density is ${ }^{9}$

$$
\begin{aligned}
P_{Q}\left(Q_{1}, Q_{2}\right) & =\psi_{0}\left(Q_{1}\right)^{2} \psi_{0}\left(Q_{2}\right)^{2} \\
& =\left(\gamma_{1} \gamma_{2} / \pi^{2}\right)^{1 / 2} \exp \left(-\gamma_{1} Q_{1}^{2}-\gamma_{2} Q_{2}^{2}\right),
\end{aligned}
$$

where $\gamma_{k}=4 \pi^{2} \nu_{k} / h$. For each of the three atom pairs in the molecule, the corresponding coefficients $\left(\mathscr{L}_{1}\right)_{i j}$ and $\left(\mathscr{L}_{2}\right)_{i j}$ transforming normal coordinates to physical dis tances can be calculated according to Eq. (4) and used to obtain the internuclear displacement coordinates $\left(x_{1}\right)_{i}$, and $\left(x_{2}\right)_{i j}$ of Eq. (5). In the following, we shall delete the subscripts $i$ and $j$ to reduce clutter but they should be understood to pertain. In the $x_{1}, x_{2}$ space, the normalized density becomes

$$
P_{x}\left(x_{1}, x_{2}\right)=\frac{\left(\gamma_{1} \gamma_{2}\right)^{1 / 2}}{\pi \mathcal{L}_{1} \mathscr{L}_{2}} \exp \left(-\frac{\gamma_{1} x_{1}^{2}}{\mathcal{L}_{1}^{2}}-\frac{\gamma_{2} x_{2}^{2}}{\mathcal{L}_{2}^{2}}\right)
$$

A final transformation with

$$
u=x_{1}+x_{2}
$$

and

$$
w=x_{1}-x_{2}
$$

carries the density function into one of particularly simple physical meaning, yielding

$$
P(u, w)=\frac{\left(\gamma_{1} \gamma_{2}\right)^{1 / 2}}{2 \pi \mathcal{L}_{1} \mathcal{L}_{2}} \exp \left(-\frac{u^{2}+w^{2}}{2 \sigma^{2}}-\beta u w\right),
$$

where

$$
\sigma^{2}=\left[\left(\gamma_{1} / 2 \mathcal{L}_{1}^{2}\right)+\left(\gamma_{2} / 2 \mathcal{L}_{2}^{2}\right)\right]^{-1}
$$

and

$$
\beta=\left[\left(\gamma_{1} / 2 \mathcal{L}_{1}^{2}\right)-\left(\gamma_{2} / 2 \mathcal{L}_{2}^{2}\right)\right] .
$$

Note that $u$ is just $\Delta r$, and that, along a line of constant $u$, the internuclear distance

$$
r=r_{\theta}+\left(x_{1}+x_{2}\right)=r_{e}+u
$$

is also constant, irrespective of the value of $w$. Therefore, in order to determine the probability $p_{i j}(u) d u$ $=p_{i j}(\Delta r) d r$ that the internuclear distance is between $r$ and $r+d r$, it is only necessary to consider contours of constant $r$ in the $u, w$ density map and sum over the elements of probability along the $w$ direction in the strip between $u$ and $u+d u$, obtaining

$$
\begin{aligned}
p_{i j}(u) d u & =\left(\int_{-\infty}^{\infty} P(u, w) d w\right) d u \\
& =\left(\gamma_{1} \gamma_{2} / 2 \pi\right)^{1 / 2}\left(\sigma / \mathfrak{L}_{1} \mathcal{L}_{2}\right) \exp \left(-u^{2} / 2 l_{i j}^{2}\right) d u,
\end{aligned}
$$

where the mean square amplitude of vibration $l_{i}^{2}$ associated with the $i j$ atom pair is

$$
l_{i j}^{2}=\sigma^{2} /\left(1-\beta^{2} \sigma^{4}\right) \text {. }
$$

By this method, we have reduced a two-dimensional density to a one-dimensional radial distribution function. A procedure for extending such reductions to a system with an arbitrary number of normal modes, each with arbitrary quantum number, is outlined in the next section.

\section{Reduction of $\mathrm{N}$-dimensional density function}

Rewriting Eq. (2) more explicitly, we get for the probability density corresponding to an arbitrary excited state of a polyatomic oscillator the expression ${ }^{9}$

$$
P_{Q}\left(Q_{1}, \ldots, Q_{N}\right)=\prod_{k} N_{k}^{2}\left[H_{v k}\left(\gamma_{k}^{1 / 2} Q_{k}\right)\right]^{2} \exp \left(-\gamma_{k} Q_{k}^{2}\right)
$$

where $N_{k}=\left[(\pi / \gamma)^{1 / 2} 2^{v k}\left(v_{k} !\right)\right]^{-1 / 2}$ and $H_{v k}$ is the Hermite polynomial of degree $v_{k}$. Reduction to the one-dimensional radial distribution function proceeds as in the previous section. For each atom pair in the molecule, the $\left(\mathscr{L}_{k}\right)_{i j}$ coefficients relating the normal coordinates to the internuclear distance $r_{i j}$ [cf. Eq. (3)] can be calculated by means of Eq. (4). With their aid $P_{Q}\left(Q_{1}, \ldots, Q_{N}\right)$ is transformed into the normalized probability density

$$
\begin{aligned}
P_{x}\left(x_{1}, \ldots, x_{N}\right) & =\prod_{k}\left(\frac{N_{k}^{2}}{\mathcal{L}_{k}}\right)\left[H_{v k}\left(\frac{\gamma_{k}^{1 / 2} x_{k}}{\mathcal{L}_{k}}\right)\right]^{2} \exp \left(-\frac{\gamma_{k} x_{k}^{2}}{\mathcal{L}_{k}^{2}}\right) \\
& \equiv \prod_{k} P_{k}\left(x_{k}\right)
\end{aligned}
$$

Reduction is carried out in steps, each step following the procedure outlined in Sec. IIC. Step (1), for example, transforms $x_{1}$ and $x_{2}$ to $u_{1}$ and $w_{1}$, via Eqs. (8a) and (8b), respectively, and the product $P_{1}\left(x_{1}\right) \cdot P_{2}\left(x_{2}\right)$ of Eq. (15) is reduced to $p_{1}\left(u_{1}\right)$ by the integration

$$
p_{1}\left(u_{1}\right)=\int_{-\infty}^{\infty} P\left(u_{1}, w_{1}\right) d w_{1}
$$

analogous to that of Eq. (12), where $P\left(u_{1}, w_{1}\right)$ is given by

$$
\frac{N_{1}^{2} N_{2}^{2}}{2 \mathcal{L}_{1} \mathcal{L}_{2}}\left[H_{v 1}\left(\frac{\gamma_{1}^{1 / 2}\left[u_{1}+w_{1}\right]}{2 \mathcal{L}_{1}}\right) H_{v 2}\left(\frac{\gamma_{2}^{1 / 2}\left[u_{1}-w_{1}\right]}{2 \mathcal{L}_{2}}\right)\right]^{2} e^{-\eta_{1}},
$$

with $\eta_{1}=\left\{\left[\left(u_{1}^{2}+w_{1}^{2}\right) / 2 \sigma_{1}^{2}\right]+\beta_{1} u_{1} w_{1}\right\}$, where the subscripts of $u, w, \sigma$, and $\beta$ denote the reduction step. An evaluation of $p_{1}\left(u_{1}\right)$, then, is expressible as the sum of integrals of the form

$$
M_{n}\left(u_{1}\right)=\int_{-\infty}^{\infty} w_{1}^{n} \exp \left[-\left(w_{1}^{2} / 2 \sigma_{1}^{2}\right)-\beta_{1} u_{1} w_{1}\right] d w_{1},
$$

which are readily evaluated analytically, ${ }^{11}$ where $n$ is an integer between 0 and $2\left(v_{1}+v_{2}\right)$. 

by

In the next step, the product $p_{1}\left(u_{1}\right) P_{3}\left(x_{3}\right)$ is transformed

$$
u_{2}=u_{1}+x_{3}
$$

and

$$
w_{2}=u_{1}-x_{3}
$$

to yield $P\left(u_{2}, w_{2}\right)$. This two-dimensional function is reduced to the distribution $p_{2}\left(u_{2}\right)$ by integration over the coordinate $w_{2}$. Subsequently, the product $p_{2}\left(u_{2}\right) P_{4}\left(x_{4}\right)$ is transformed to $P\left(u_{3}, w_{3}\right)$ from which $p_{3}\left(u_{3}\right)$ is obtained, and so on. For reduction steps beyond the first, the amplitude parameters $\sigma_{m}^{2}, \beta_{m}$, and $\eta_{m}$ take on the values

$$
\begin{aligned}
& \sigma_{m}^{2}=\left(\frac{1}{4 \sigma_{m-1}^{2}}+\frac{\gamma_{k}}{2 \mathcal{L}_{k}^{2}}\right)^{-1}, \\
& \beta_{m}=\left(\frac{1}{4 \sigma_{m-1}^{2}}-\frac{\gamma_{k}}{2 \mathcal{L}_{k}^{2}}\right),
\end{aligned}
$$

and

$$
\eta_{m}=\left\{\left[\left(u_{m}^{2}+w_{m}^{2}\right) / 2 \sigma_{m}^{2}\right]+\beta_{m} u_{m} w_{m}\right\},
$$

respectively, in which the subscript $m$ denotes the reduction step and the subscript $k$ denotes the new mode to be included in the reduction. Recursion formulas (20a) and (20b) apply to steps with $m \geq 2$. In the case of $m=1$, the parameters $\sigma_{1}^{2}$ and $\beta_{1}$ are given by Eqs. (10a) and (10b), respectively. Reductions are carried out until the last $x_{k}$ has been taken into account, and the resultant $p_{N}\left(u_{N}\right)$ is the desired radial distribution function for the atom pair $i, j$ inasmuch as $u_{N}=\sum_{k} x_{k}$ is identically $\Delta r_{i j}$

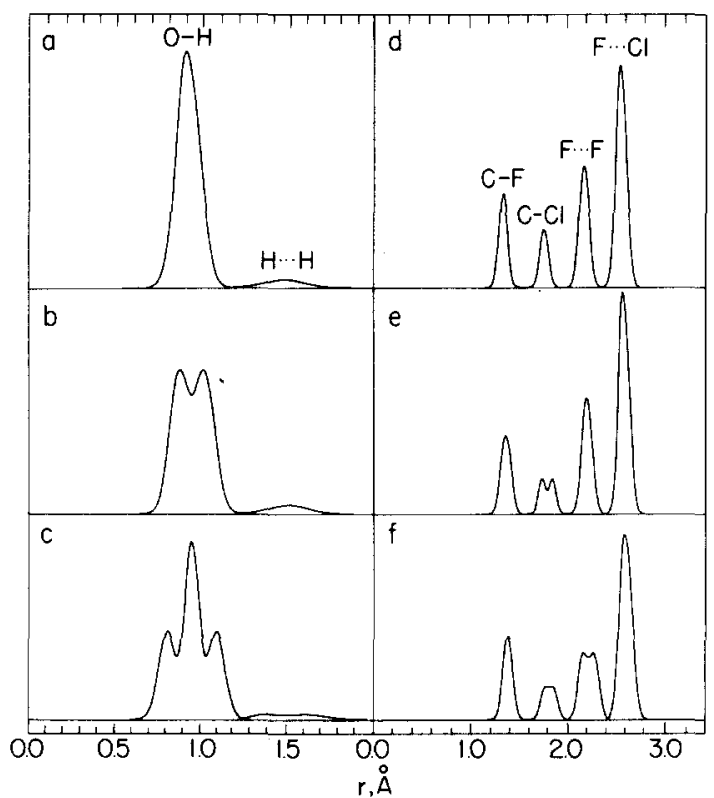

FIG. 1. Molecular radial distribution functions calculated according to Eq. (22) of the text. $\mathrm{H}_{2} \mathrm{O}$ : (a) zero-point amplitudes of vibration; (b) single excitation of asymmetric stretch $(0,0,1)$; (c) single excitation of both stretching modes $(1,0,1) . \mathrm{CF}_{3} \mathrm{Cl}$ : (d) zero-point amplitudes of vibration; (e) single excitation of the $\nu_{1}$ mode $(1,0,0,0,0,0)$ customarily referred to as the $\mathrm{C}-\mathrm{F}$ symmetric stretch but principally manifested in the $\mathrm{C}-\mathrm{Cl}$ distribution; (f) double excitation of the $\nu_{2}$ mode $(0,2,0,0,0,0)$ customarily described as the $\mathrm{C}-\mathrm{Cl}$ stretch but predominantly exhibited in the $\mathrm{F}-\mathrm{C}-\mathrm{F}$ bend $(\mathrm{F} \cdots \mathrm{F}$ peak).

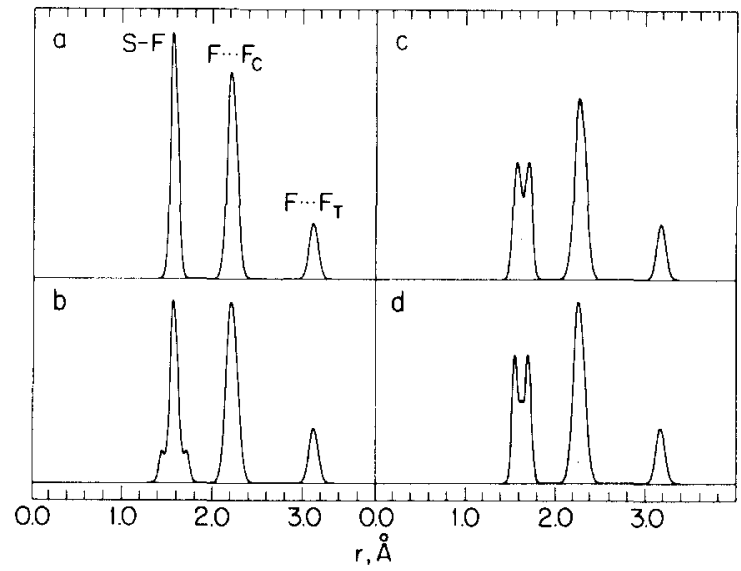

FIG. 2. Molecular radial distribution functions of $\mathrm{SF}_{6}$ calculated according to Eq. (22) of text: (a) zero-point amplitudes of vibration. Remaining curves, six quanta distributed among triply degenerate $\nu_{3}$ asymmetric stretching modes $\left(v_{3 a}, v_{3 b}\right.$, $\left.v_{3 c}\right)$ : (b) $(6,0,0)$; (c) $(3,2,1)$; (d) $(2,2,2)$.

by virtue of Eq. (5). The final result does not depend upon the order in which the individual $x_{k}$ are reduced but it is simpler to begin with the modes of lowest quantum number and finish with the highest. This is because index $n$ of integral $M_{n}$ of Eq. (18) increases each time an excited mode is brought into the reduction, thus proliferating the numbers of terms to be handled in all subsequent steps.

If comparisons are to be made with electron diffraction studies, it may be convenient to calculate a composite radial distribution function $p(r)$ for the entire molecule in which the individual internuclear peaks are weighted by the relative scattering powers, or

$$
p(r)=2 \sum_{i>j}\left\{Z_{i} Z_{j} /\left[\sum_{k}\left(Z_{k}^{2}+Z_{k}\right)\right]\right\} p_{i j}(r) .
$$

Sometimes it may be desirable to transform Eq. (22) into the so-called $f(r)$ function corresponding to the Fourier sine transform of the modified experimental intensity function $s M(s)$ artificially damped by the Degard factor $\exp \left(-b s^{2}\right)$. The result is, ${ }^{12,13}$ in the notation of Ref. 13,

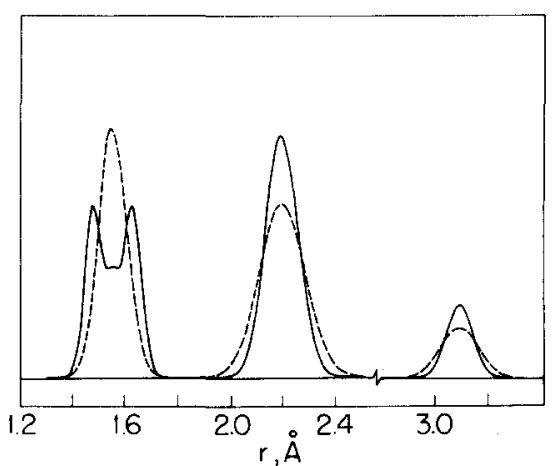

FIG. 3. Molecular radial distribution peaks in $\mathbf{S F}_{6}$. Solid line, six quanta in $\nu_{3}$ distributed as in Fig. 2(d). Dashed line, same vibrational energy distributed among all modes according to Boltzmann distribution $(T=911.5 \mathrm{~K})$. 
TABLE I. Root-mean-square amplitudes of vibration (in $\mathscr{A}$ ) corresponding to the radial distribution functions of $\mathrm{H}_{2} \mathrm{O}$ and $\mathrm{CF}_{3} \mathrm{Cl}$ shown in Fig. 1 .

\begin{tabular}{|c|c|c|c|}
\hline \multirow[b]{2}{*}{ Peak } & \multicolumn{3}{|c|}{ Curve } \\
\hline & 1(a) & $1(b)$ & $1(c)$ \\
\hline $\mathrm{O}-\mathrm{H}$ & 0.06770 & 0.09571 & 0.1171 \\
\hline \multirow[t]{2}{*}{$\mathrm{H} \cdots \mathrm{H}$} & 0.1114 & 0.1114 & 0.1545 \\
\hline & \multicolumn{3}{|c|}{ Curve } \\
\hline Peak & 1 (d) & $1(\mathrm{e})$ & $1(\mathrm{f})$ \\
\hline $\mathrm{C}-\mathrm{F}$ & 0.04431 & 0.05122 & 0.04948 \\
\hline $\mathrm{C}-\mathrm{Cl}$ & 0.04495 & 0.06590 & 0.06444 \\
\hline$F \cdots F$ & 0.05149 & 0.05392 & 0.07794 \\
\hline $\mathrm{F} \cdots \mathrm{Cl}$ & 0.05322 & 0.05347 & 0.06237 \\
\hline
\end{tabular}

$f_{c}(\gamma)=(\pi / 16 b)^{1 / 2} \int_{-\infty}^{\infty} \rho^{-1} p(\rho) \exp \left[-(r-\rho)^{2} / 4 b\right] d \rho$.

If molecules in an ensemble populate a distribution of vibrational states, the $p(r)$ and $f(r)$ curves for the ensemble are, of course, simple superpositions of curves calculated for the individual states represented.

\section{ILLUSTRATIVE RESULTS}

In order to illustrate how radial distribution curves vary from vibrational state to vibrational state-and to convey graphically how broad the distribution peaks can be-we portray in Figs. 1-3 some curves calculated ${ }^{14}$ according to Eq. (22). Root-mean-square amplitudes of vibration of the individual distribution peaks plotted in Figs. 1-3 are listed in Tables $\mathrm{I}$ and II for reference. Molecular examples include $\mathrm{H}_{2} \mathrm{O}, \mathrm{CF}_{3} \mathrm{Cl}$, and $\mathrm{SF}_{6}$. A case such as water is simple enough for the details of its dominant peak $(\mathrm{OH})$ to be understood intuitively from a visualization of the two dimensional probability density function $P_{1}\left(x_{1}\right) P_{3}\left(x_{3}\right)$ for $\mathrm{OH}$ stretching. Therefore, it is a helpful conceptual bridge between elementary onedimensional oscillators and multidimensional oscillators. A more complex, nine-dimensional oscillator with six distinct frequencies is $\mathrm{CF}_{3} \mathrm{Cl}$, selected because of the particularly distinct resolution of its four internuclear peaks. A final example is $\mathrm{SF}_{6}$, the subject of intense current interest in laser-induced processes. ${ }^{2}$ For sake of illustration, we show in Figs. 2 and 3 the effect of various excitations of the $t_{1 s}$ stretching modes, the modes directly pumped by a $\mathrm{CO}_{2}$ laser. A comparison is made with thermally excited $\mathrm{SF}_{6}$ possessing the same mean vibrational energy.

It is apparent in the foregoing illustrations that a precise measurement of radial distribution functions of ensembles of molecules in excited states would yield useful but incomplete information about the distribution of vibrational energy in the molecules. Since measurements by electron diffraction can be made at controlled
TABLE II. Root-mean-square amplitudes of vibration (in $\AA$ ) corresponding to the radial distribution functions of $\mathrm{SF}_{6}$ shown in Fig. 2.

\begin{tabular}{lcccc}
\hline \hline & \multicolumn{4}{c}{ Curve } \\
\cline { 2 - 5 } Peak & $2(\mathrm{a})$ & $2(\mathrm{~b})$ & $2(\mathrm{c})$ & $2(\mathrm{~d})$ \\
\hline $\mathrm{S}-\mathrm{F}$ & 0.04100 & 0.07613 & 0.07613 & 0.07613 \\
$\mathrm{~F} \cdots \mathrm{F}_{c}$ & 0.05530 & 0.06262 & 0.06262 & 0.06262 \\
$\mathrm{~F} \cdots \mathrm{F}_{T}$ & 0.05107 & 0.05107 & 0.05107 & 0.05107 \\
\hline \hline
\end{tabular}

time delays, of the order of microseconds after the irradiation of samples, it is possible that diffraction measurement may yield information on the rate of energy redistribution in the case of slow $v-v$ relaxation. The foregoing treatment may be of value in such studies.

\section{ACKNOWLEDGMENTS}

This research was supported by a grant from the National Science Foundation. We thank Dr. E. J. Jacob for valuable suggestions about numerical calculations. We gratefully acknowledge a generous allotment of computing time from the Michigan Computing Center.

${ }^{1}$ See, for example, I. Burak, J. I. Steinfeld, and D. G. Sutton, J. Quant. Spectrosc. Radiat. Transfer 9, 959 (1969); R. D. Bates, J. T. Knudtson, G. W. Flynn, and A. R. Ronn, Chem. Phys. Lett. 8, 103 (1971).

${ }^{2}$ See, for example, N. Bloembergen and E. Yablonovitch, Phys. Today 31, 23 (1978); R. V. Ambartzumian and V. S. Letokhov, Acc. Chem. Res. 10, 61 (1977); E. Grunwald, K. J. Olszyna, D. F. Dever, and B. Knishkowy, J. Am. Chem. Soc. 99, 6515 (1977).

${ }^{3}$ Y. Morino and T. Iijima, Bull. Chem. Soc. Jpn. 36(4), 412 (1963).

${ }^{4}$ See the references in S. J. Cyvin, Molecular Vibrations and Mean Square Amplitudes (Elsevier, Amsterdam, 1968).

${ }^{5}$ L. S. Bartell, J. Chem. Phys. 23, 1219 (1955).

${ }^{6} \mathrm{For}$ treatments illustrating the effect of nonlinear transformations and the influence of anharmonicity upon wave functions of polyatomic molecules, see, for example, K. Kuchitsu and L. S. Bartell, J. Chem. Phys. 36, 2460, 2470 (1962).

${ }_{7}$ M. Mills, J. Phys. Chem. 80, $1187(1976)$.

${ }^{8}$ L. S. Bartell and S. Fitzwater, J. Chem. Phys. 67, 4168 (1977).

${ }^{9}$ E. B. Wilson, Jr., J. C. Decuis, and P. C. Cross, Molecular Vibrations (McGraw-Hill, New York, 1955).

${ }^{10}$ B. L. Crawford, Jr. and W. H. Fletcher, J. Chem. Phys. 19, 141 (1951); Y. Morino and E. Hirota, J. Chem. Phys. 23, 737 (1955); see also Ref. 5 .

${ }^{11}$ I. S. Gradshteyn and I. M. Ryzhik, Tables of Integrals Series and Products (Academic, New York, 1965), 4th edition, p. 337.

${ }^{12}$ I. L. Karle and J. Karle, J. Chem. Phys. 17, 1052 (1949).

${ }^{13}$ R. A. Bonham and L. S. Bartell, J. Chem. Phys. 31, 702 (1959).

${ }^{14}$ Force Fields for $\mathrm{H}_{2} \mathrm{O}, \mathrm{CF}_{3} \mathrm{Cl}$, and $\mathrm{SF}_{6}$ were taken from $\mathrm{L}$. S. Bartell and K. Kuchitsu, J. Chem. Phys. 37, 691 (1962); R. C. Taylor, J. Chem. Phys. 22, 714 (1954); B. Weinstock and G. Goodman, Adv. Chem. Phys. 9, 309 (1965). 